\title{
Amostragem para diagnose do estado nutricional e avaliação da fertilidade do solo em caramboleiras
}

\author{
Amanda Hernandes $\left({ }^{*}\right)$; Danilo Eduardo Rozane $\left({ }^{2}\right)$; Henrique Antunes de Souza $\left({ }^{3}\right)$; \\ Liliane Maria Romualdo ( $\left.{ }^{4}\right)$; William Natale ( $\left.{ }^{1}\right)$ \\ (') Universidade Estadual Paulista Júlio de Mesquita Filho (UNESP), Faculdade de Ciências Agrárias e Veterinárias (FCAV), \\ Departamento de Solos e Adubos. Via de Acesso Prof. Paulo Donato Castellane, s/n, 14884-900 Jaboticabal (SP). \\ (2) UNESP, Campus Experimental de Registro (FCAVR), Rua Nelson Brihi Badur, 430, 11900-000 Registro (SP). \\ (3) Embrapa Caprinos e Ovinos, Caixa Postal 145, 62011-970 Sobral (CE). \\ (4) Universidade de São Paulo (USP), Faculdade de Zootecnia e Engenharia de Alimentos, 13635-900 Pirassununga (SP). \\ (*) Autora correspondente: amahernandes@hotmail.com
}

Recebido: 10/ago./2010; Aceito: 2/mar./2011

\begin{abstract}
Resumo
Para a realização de uma análise confiável, a etapa mais sensível e que merece extremo cuidado é a amostragem do tecido vegetal e do solo. A amostra mais adequada é aquela que representa o melhor possível a área de estudo, exigindo um mínimo de plantas amostradas para atender a esse objetivo e com o menor número possível de amostras simples coletadas. Assim, o presente trabalho procurou dimensionar o número de plantas a serem amostradas para a diagnose do estado nutricional, bem como o número de amostras simples necessárias para formar a amostra composta, para fins de avaliação da fertilidade do solo cultivado com caramboleiras. O estudo foi realizado em um pomar comercial de caramboleiras, no município de Vista Alegre do Alto (SP), empregando-se amostragem aleatória, coletando-se a sexta folha a partir do ápice do ramo da caramboleira, na altura mediana da frutífera, no florescimento da cultura, em 40 plantas. Foram coletadas, também, 30 amostras simples de solo, em zigue-zague, nas linhas da cultura, com o auxílio de um trado tipo holandês, nas camadas de 0 a 0,2 m e 0,2 a 0,4 m. Considerando-se aceitável um erro amostral de 10\%, 21 plantas de carambola seriam suficientes para as determinações químicas foliares de macronutrientes. Já para os micronutrientes, seriam necessárias, no mínimo, 52 plantas amostradas. O aumento do número de amostras simples reduziu o erro porcentual na estimativa da média desejada, permitindo a recomendação de 14 e 17 amostras simples nas camadas de 0 a 0,2 m e 0,2 a 0,4 m (erro = 20\%), respectivamente.
\end{abstract}

Palavras-chave: Averrhoa carambola, amostragem de folhas, amostragem de solo, nutrição mineral, carambola, frutífera.

\section{Sampling for diagnosis of nutritional status and assessment of fertility in starfruit orchards}

\section{Abstract}

For a reliable nutritional analysis, the most sensitive stage is the plant and soil samplings. The most proper sampling is the one that best represents the area under study, requiring a minimum of sampled plants and the least possible number of simple samples collected. This work aimed to assess the number of plants to be sampled for the diagnosis of nutritional status, as well as the number of simple samples for making up of the compost sample for evaluating the soil fertility in starfruit orchard. This study was performed in a commercial starfruit orchard, in Vista Alegre do Alto, State of São Paulo. Random sampling was taken, collecting the sixth leaf from the apex of the branch of 40 starfruit trees, at the medium canopy height during blooming season. In the same way, 30 simple soil samples were collected, in a zigzag pattern following the planting lines, using a Dutch-type auger, at depths of 0 to $0.2 \mathrm{~m}$ and 0.2 to $0.4 \mathrm{~m}$. Assuming a sample error of 10\%, 21 starfruit trees would be sufficient for determining the chemical leaf macronutrients. For micronutrients, a minimum of 52 sampled plants would be necessary. Increasing the number of soil samples resulted in reduced percentage of error within the desired average estimate, thus allowing for a recommendation of 14 to 17 simple samples at depths of 0 to $0.2 \mathrm{~m}$ and 0.2 to $0.4 \mathrm{~m}$ (error = 20\%), respectively.

Key words: Averrhoa carambola, leaf sampling, soil sampling, mineral nutrition, starfruit, fruit tree. 


\section{INTRODUÇÃO}

Em caramboleiras, a análise química de folhas auxilia na determinação do estado nutricional da cultura, avalia e calibra o resultado das adubaçôes realizadas com base na interpretação da análise de solo e, ainda, é empregada para estabelecer a recomendação da adubação nitrogenada. MarsChNer (1995) ressalta que, para frutíferas, a análise foliar se torna ainda mais útil que a análise de solo.

Um dos principais condicionantes ao êxito da correta interpretação do estado nutricional das plantas está na amostragem, em especial quanto ao número de plantas a serem amostradas por talhão, para se ter confiabilidade na diagnose.

A indicação mais aceita para a época de amostragem de folhas da caramboleira é no pleno florescimento do pomar (Prado e Natale, 2004), coletando-se a sexta folha composta. Crane (1998) indica que devem ser amostradas no mínimo 10 árvores por talhão homogêneo e no mínimo 10 folhas por árvore, coletadas em todos os lados da planta.

A importância do número de árvores a serem amostradas para representar adequadamente uma população (ou pomar), foi objeto de pesquisa na cultura da maçã (Holland et al., 1967), do eucalipto (Lamb, 1976), do cajueiro (Rossetti et al., 1991), em plantaçôes de arbóreas (MeAd, 1984; Rossetti et al., 1996), em mangueiras (RozAne et al., 2007) e em goiabeiras (RozAne et al., 2009); entretanto, não há estudos com a cultura da carambola, considerando-se este aspecto.

O problema da representatividade de amostras para determinar a fertilidade do solo está na desuniformidade espacial dos atributos químicos, ou seja, na variabilidade do solo. As diferenças nas características químicas do solo ocorrem naturalmente, como consequências de processos pedogenéticos (Mausbach e Wilding, 1991), manifestando-se nas direçóes horizontal e vertical do solo, podendo ser alteradas, também, pelas açōes antrópicas, mediante o manejo da área (James e Wells, 1990).

$\mathrm{Na}$ avaliação da fertilidade do solo a amostragem é, sem dúvida, a etapa mais sensível e sujeita a erros. Sabe-se que, do erro total que advêm de um resultado analítico, 80\%-85\% são atribuídos à amostragem no campo, e apenas 15\%-20\% aos procedimentos de laboratório (EMBRAPA, 1999a; Montesinos et al., 2002).

A adequada representatividade da área, por meio da amostra composta, está diretamente relacionada ao cuidado na amostragem (GUARÇONI et al., 2006). As amostras simples devem ser coletadas à mesma profundidade, possuir o mesmo volume e refletir as condiçóes de fertilidade de seus respectivos sítios de coleta.

A utilização do trado tem como vantagem a rapidez na coleta das amostras simples, além do manuseio e transporte de menor volume de solo no campo, antes da homogeneização para a retirada da amostra composta
(Oliveira et al., 2007). Porém, o menor volume de solo coletado com o trado faz com que a variabilidade dos índices de fertilidade do solo aumente, tornando necessário coletar maior número de amostras simples para formar uma amostra composta que seja representativa da área. Como alternativa, Alvarez e Guarçoni (2003) sugerem que, sob sistema de plantio direto, a variabilidade do trado poderia ser contornada se, durante a amostragem de solo após a colheita e antes do subsequente revolvimento do solo, $17 \%$ das amostras simples fossem coletadas no sulco de plantio, $33 \%$ a $10 \mathrm{~cm}$ do sulco e $50 \%$ no ponto médio entre os sulcos.

Para solos tropicais, RAIJ et al. (1997) recomendam a coleta de 20 amostras simples para compor uma amostra composta. Porém, em inúmeros trabalhos realizados, não há concordância quanto ao número de amostras necessárias para se estimar a fertilidade média, ocorrendo variaçôes bastante significativas. Em estudos mais recentes, considerando-se o atributo mais limitante e o erro porcentual na estimativa da média de $20 \%$, o número de amostras simples pode variar de 50 a 188 (Silveira et al., 2000); de 11 a 183 (Schlindwein e Anghinoni, 2002); de 20 a 39 (Guarçoni et al., 2006); de 5 a 20 (GuARÇONi et al., 2007), e de 18 a 31 (Amaro Filho et al., 2007).

De maneira geral, pode-se afirmar que as amostras de solo e de tecido vegetal mais adequadas sáo aquelas que representam o melhor possível a área a ser avaliada, com o menor número possível de amostras simples de solo coletadas e um mínimo de plantas amostradas para atender a esse objetivo. Assim, a análise estatística auxilia na indicação do número mínimo de pontos no solo e árvores suficientes para reduzir a variação dos resultados a um nível aceitável, ou seja, reduzindo o número de graus de liberdade que exprimem o acaso. Com isso, tem-se uma amostra representativa da área e da população de plantas para que os resultados da análise de solo e de tecido foliar tenham validação técnica e científica, e que recursos não sejam empregados desnecessariamente, evitando-se amostragens não representativas.

Buscando-se maiores esclarecimentos sobre o assunto objetivou-se, neste estudo, dimensionar o número de amostras simples de solo necessárias para formar a amostra composta, para avaliaçáo da fertilidade do solo, bem como o número de plantas a serem amostradas para a diagnose do estado nutricional da caramboleira.

\section{MATERIAL E MÉTODOS}

O experimento foi realizado em um pomar comercial de caramboleiras irrigadas, de oito anos, com 700 plantas em 2,5 ha, localizado no município de Vista Alegre do Alto (SP), com coordenadas geográficas $21^{\circ} 15^{\prime} 22^{\prime \prime}$ Sul, $48^{\circ}$ 18' 58" Oeste e altitude de $603 \mathrm{~m}$. Segundo a classificação de Köppen, o clima local é do tipo Cwa subtropical com 
inverno curto, moderado e seco, verão quente e chuvoso, caracterizando duas estaçôes distintas. O solo do pomar foi classificado como Latossolo Vermelho-Amarelo distrófico, textura arenosa/média, correspondendo ao Latossolo Vermelho-Amarelo distrófico, segundo a Embrapa (1999b).

Para a diagnose do estado nutricional das caramboleiras, coletou-se a sexta folha a partir do ápice do ramo, na altura mediana das plantas, sendo uma em cada um dos quatro pontos cardeais da frutífera, em pleno florescimento (Prado e Natale, 2004), em 40 plantas. Os teores de macro e micronutrientes foram determinados seguindo método descrito por Bataglia et al. (1983). Com base nos resultados dos teores foliares de nutrientes, foram calculadas as médias, as variâncias, os erros-padrão das médias, o intervalo de confiança para as médias e as porcentagens de erro em relação às médias, através da semiamplitude do intervalo de confiança expresso em porcentagem da média.

Para avaliar a fertilidade do solo, foram coletadas 30 amostras simples, em zigue-zague, de maneira aleatória, nas linhas da cultura, com o auxílio de um trado tipo holandês, nas camadas de 0-0,20 m e 0,20-0,40 m.

As determinaçóes analíticas $\left(\mathrm{pH}\right.$ em $\mathrm{CaCl}_{2}, \mathrm{P}_{\text {resina' }}$, M.O., K, Ca, Mg e H+Al) e o parâmetro calculado (V\%) seguiram os métodos descritos por RaIj et al. (2001). Para cada atributo foi calculada a média, o desvio-padrão, o erro-padrão da média e o coeficiente de variação.

Tanto para a amostragem de folhas como para a amostragem de solo, o número de unidades amostrais para estimar os parâmetros de uma população infinita para um nível de precisão desejado, baseando-se no erro-padrão da média, foi dado pela equação descrita em THOMPson (1992):

$$
n=\frac{t^{2} s^{2}}{d^{2} m^{2}}
$$

Na qual: $n$ é o tamanho da amostra estimada; $t$ é o valor da distribuição $t$ de Student a $5 \%$ de probabilidade; $s^{2}$ é a variância; $d$ é o erro na estimativa da média, em porcentagem, variando de $1 \%$ a $30 \%$ de $m$; $m$ é a média amostral.

\section{RESULTADOS E DISCUSSÃO}

Os teores de macro e micronutrientes nas folhas de caramboleira estão, de maneira geral, dentro da faixa recomendada (Tabela 1), conforme dados apresentado por DonAdio et al. (2001). Os maiores erros amostrais foram detectados quando se coletaram amostras em menor número de plantas, diminuindo o erro com o aumento do número de caramboleiras amostradas (Tabela 1).

Para florestas de eucalipto, LAMB (1976) propóe que um erro entre $5 \%$ e $10 \%$ seria aceitável, indicando ser necessário amostrar 10 plantas quando se quer analisar apenas os macronutrientes, mas 20 árvores quando se de- seja analisar, também, os micronutrientes. MeAd (1984) considera para Pinus radiata um erro aceitável entre 5\% e $10 \%$ para a maioria dos macronutrientes e o $\mathrm{Cu}$; mas para o Ca e os demais micronutrientes é normal que se tenham erros maiores, decorrentes do maior coeficiente de variação entre árvores. KNight (1978) considera que grande parte da discrepância nos teores foliares em Pinus está associada à diferença clonal, embora essa grandeza variasse menos de $2 \%$ para o $\mathrm{Ca}$, mas $48 \%$ para o $\mathrm{B}$.

Para o diagnóstico foliar, MEAD (1984) recomenda coletar amostras em 20 árvores em florestas de eucalipto. Rozane et al. (2007), admitindo como erro aceitável de $5 \%$ a $10 \%$, recomendam a amostragem de 10 plantas de mangueiras para a determinação de macronutrientes e, no mínimo 30 plantas, para a análise de micronutrientes. RozAne et al. (2009), considerando a mesma porcentagem de erro (5\% a $10 \%)$, em estudo em pomar adulto de goiabeiras, cultivar Paluma, indicam como suficiente a amostragem de 10 plantas para macronutrientes e 40 plantas para a determinação dos micronutrientes.

As amplas variaçóes ocorridas nos teores foliares de $\mathrm{Cu}, \mathrm{Mn}, \mathrm{Fe}$ e $\mathrm{Zn}$ são comuns neste tipo de análise, também relatadas por LAmb (1976) em eucalipto, por Dahiya e Joon (1995) e por Rozane et al. (2009) em goiabeira, por Sodré et al. (2001) em cacaueiro, e por Rozane et al. (2007) em mangueiras. As oscilaçóes nas análises foliares podem ser decorrentes das pulverizaçôes foliares à base de cobre e zinco, normalmente realizadas em pomares comerciais. Além disso, no caso do ferro e do manganês, o tipo de solo pode ser determinante, visto que em condiçóes de acidez (normalmente observada no Brasil) esses elementos tornam-se mais disponíveis. Assim, na presença de concentraçóes excessivas desses micronutrientes no meio de crescimento, os tecidos vegetais apresentarão, também, teores altos, visto que as plantas absorvem e transportam os elementos em quantidades elevadas, resultando em acúmulo nas folhas, podendo atingir até níveis tóxicos (Foy, 1973; Pavan e Bingham, 1981).

Admitindo-se um erro aceitável de 5\% a 10\%, 21 plantas de caramboleira foram suficientes para a análise dos macronutrientes (Tabela 1). Porém, para os micronutrientes, foram necessárias, no mínimo, 52 plantas para manter o erro menor que $10 \%$.

A variabilidade de um atributo pode ser classificada de acordo com a magnitude do seu coeficiente de variação, que pode ser: (a) baixa, quando menor que 10\%; (b) média, quando entre $10 \%$ e $20 \%$; (c) alta, quando entre $20 \%$ e $30 \%$; e (d) muito alta, se maior que 30\% (PIMENtel-Gomez e Garcia, 2002). Assim, de modo geral, no presente trabalho, os atributos $\mathrm{pH}$ e V\% tiveram variabilidade baixa; os atributos $\mathrm{MO}$ e $\mathrm{K}$, variabilidade média; $\mathrm{Ca}$ e $\mathrm{Mg}$, variabilidade alta; e o atributo $\mathrm{P}$, variabilidade muito alta (Tabela 2). De maneira geral, baixos teores do componente fazem com que pequenas alteraçôes gerem altos coeficientes de variação. 
Tabela 1. Estatística descritiva e estimativa do número de plantas necessárias para avaliação dos teores de macro e micronutrientes em caramboleiras, em função do erro na estimativa da média

\begin{tabular}{|c|c|c|c|c|c|c|}
\hline \multirow[t]{2}{*}{ Estimativa da média "f" } & \multicolumn{6}{|c|}{ Macronutrientes $\left(\mathbf{g ~ k g}^{-1}\right)$} \\
\hline & $\mathbf{N}$ & $\mathbf{P}$ & K & $\mathrm{Ca}$ & Mg & $\mathbf{S}$ \\
\hline 1 & 436 & 560 & 708 & 2114 & 1586 & 632 \\
\hline 5 & 17 & 22 & 28 & 85 & 63 & 25 \\
\hline 10 & 4 & 6 & 7 & 21 & 16 & 6 \\
\hline 15 & 2 & 3 & 3 & 9 & 7 & 3 \\
\hline 20 & 1 & 1 & 2 & 5 & 4 & 2 \\
\hline 30 & 0 & 1 & 1 & 2 & 2 & 1 \\
\hline 40 & 0 & 0 & 0 & 1 & 1 & 0 \\
\hline$\hat{m}$ & 22,03 & 1,14 & 14,42 & 11,95 & 5,62 & 1,47 \\
\hline$s$ & 1,79 & 0,10 & 1,49 & 2,14 & 0,87 & 0,14 \\
\hline$s(\hat{m})$ & 0,80 & 0,05 & 0,67 & 0,96 & 0,39 & 0,06 \\
\hline \multirow[t]{3}{*}{$\mathrm{CV}$} & 8,1 & 9,2 & 10,4 & 17,9 & 15,5 & 9,8 \\
\hline & \multicolumn{6}{|c|}{ Micronutrientes $\left(\mathrm{mg} \mathrm{kg}^{-1}\right)$} \\
\hline & B & $\mathrm{Cu}$ & $\mathrm{Fe}$ & Mn & \multicolumn{2}{|c|}{ Zn } \\
\hline 1 & 1397 & 5188 & 4057 & 4613 & \multicolumn{2}{|c|}{2985} \\
\hline 5 & 56 & 208 & 162 & 185 & \multicolumn{2}{|c|}{119} \\
\hline 10 & 14 & 52 & 41 & 46 & \multicolumn{2}{|c|}{30} \\
\hline 15 & 6 & 23 & 18 & 21 & \multicolumn{2}{|c|}{13} \\
\hline 20 & 4 & 13 & 10 & 12 & \multicolumn{2}{|c|}{7} \\
\hline 30 & 2 & 6 & 5 & 5 & \multicolumn{2}{|c|}{3} \\
\hline 40 & 1 & 3 & 3 & 3 & \multicolumn{2}{|c|}{2} \\
\hline$\hat{m}$ & 26,10 & 4,50 & 53,75 & 371,35 & \multicolumn{2}{|c|}{21,25} \\
\hline$s$ & 3,79 & 1,26 & 13,32 & 98,12 & \multicolumn{2}{|c|}{4,52} \\
\hline$s(\hat{m})$ & 1,70 & 0,56 & 5,96 & 43,88 & \multicolumn{2}{|c|}{2,02} \\
\hline CV (\%) & 14,5 & 28,0 & 24,8 & 26,4 & \multicolumn{2}{|c|}{21,3} \\
\hline
\end{tabular}

m̂ é a estimativa da média amostral; s é o desvio-padrăo; $s(\hat{m})$ é o erro-padrão da média; e CV é o coeficiente de variaçấo.

Os coeficientes de variação foram baixos para o $\mathrm{pH}$, nas duas profundidades, variando de $2,9 \%$ a $5,6 \%$, o que evidencia a baixa variabilidade desse atributo do solo. SILVeirA et al. (2000) também observaram baixo CV em relação ao $\mathrm{pH}$, variando de $3 \%$ a 5,4\%.

$\mathrm{Na}$ camada de $0-0,20 \mathrm{~m}$, a variabilidade dos atributos avaliados, estimada pelo número de amostras simples de solo necessárias para compor uma amostra composta, seguiu a ordem: $\mathrm{pH}<\mathrm{V}<\mathrm{H}+\mathrm{Al}<\mathrm{M} . \mathrm{O} .<\mathrm{K}^{+}<\mathrm{Mg}^{2+}<$ $\mathrm{Ca}^{2+}<\mathrm{P}$. Na camada de 0,20-0,40 m a variabilidade dos atributos avaliados foi um pouco diferente, seguindo a ordem: $\mathrm{pH}<\mathrm{V}<\mathrm{M} . \mathrm{O}$. $<\mathrm{Ca}^{2+}<\mathrm{H}+\mathrm{Al}<\mathrm{Mg}^{2+}<\mathrm{K}^{+}<\mathrm{P}$.

A quantidade de unidades amostrais necessárias para formar uma amostra composta, representativa do talhão homogêneo, está condicionada ao nível de precisão desejado, definido em função dos objetivos da amostragem. Considerando a necessidade de reduzir o número de amostras simples de solo no campo, é admissível um erro de até $20 \%$ em torno da média, sem imprimir aos resultados um erro elevado em torno da verdadeira média populacional, tornando o processo econômico e tecnicamente viável (Montesinos et al., 2002; GarçONi et al., 2006; 2007; Oliveira et al., 2007; Amaro Filho et al.,
2007). Schlindwein e Anghinoni (2000) afirmam que adotar erro de $10 \%$, em condiçốes de lavoura, seria excesso de rigor para a exatidão da amostragem de solo, pois podem ocorrer ainda erros laboratoriais, que influenciam a recomendação de calagem e adubação.

A estimativa do número de amostras simples necessárias para compor a amostra composta diminuiu à medida que se aumentou o erro (Tabela 2). Dentre os atributos de fertilidade avaliados, o que estimou o maior número de amostras simples necessárias para compor a amostra composta de solo foi o P, corroborando resultados de SiLVEIRA et al. (2000), Alvarez e Guarçoni (2003) e Oliveira et al. (2007). O fósforo representou a maior variabilidade dentre os atributos avaliados necessitando, portanto, do maior número de amostras simples para compor a amostra composta (Tabela 2). Considerando que o número mínimo de subamostras de solo é diretamente proporcional ao coeficiente de variação, quanto maior o $\mathrm{CV}$, maior será o número de subamostras a serem coletadas.

Dessa forma, nas condições em que o presente estudo foi desenvolvido, admitindo-se um erro de $20 \%$ em torno da média para os parâmetros avaliados, a coleta de 14 amostras simples de solo para formar uma amostra composta 
Tabela 2. Estatística descritiva e estimativa do número de amostras simples de solo, necessárias a compor a amostra composta para avaliação dos atributos químicos do solo, em função do erro na estimativa da média

\begin{tabular}{|c|c|c|c|c|c|c|c|c|}
\hline \multirow{3}{*}{$\begin{array}{l}\text { Estimativa da } \\
\text { média "f" }\end{array}$} & \multicolumn{8}{|c|}{ Camada amostrada $(\mathrm{cm})$} \\
\hline & $0-20$ & $20-40$ & $0-20$ & $20-40$ & $0-20$ & $20-40$ & $0-20$ & $20-40$ \\
\hline & \multicolumn{2}{|c|}{$\mathrm{pH}\left(\mathrm{CaCl}_{2}\right)$} & \multicolumn{2}{|c|}{$\mathrm{MO}\left(\mathrm{g} \mathrm{dm}^{-3}\right)$} & \multicolumn{2}{|c|}{$P_{\text {resina }}\left(\mathbf{m g ~ d m}^{-3}\right)$} & \multicolumn{2}{|c|}{$\mathrm{K}\left(\mathrm{mmol}_{\mathrm{c}} \mathrm{dm}^{-3}\right)$} \\
\hline 1 & 204 & 54 & 2228 & 1696 & 5678 & 6886 & 2511 & 2913 \\
\hline 5 & 8 & 2 & 89 & 68 & 227 & 275 & 100 & 117 \\
\hline 10 & 2 & 1 & 22 & 17 & 57 & 69 & 25 & 29 \\
\hline 15 & 1 & 0 & 10 & 8 & 25 & 31 & 11 & 13 \\
\hline 20 & 1 & 0 & 6 & 4 & 14 & 17 & 6 & 7 \\
\hline 25 & 0 & 0 & 4 & 3 & 9 & 11 & 4 & 5 \\
\hline 30 & 0 & 0 & 2 & 2 & 6 & 8 & 3 & 3 \\
\hline$\hat{m}$ & 5,3 & 5,8 & 15,6 & 10,8 & 100,27 & 30,0 & 4,35 & 4,19 \\
\hline$s$ & 0,29 & 0,17 & 2,87 & 1,73 & 29,39 & 9,68 & 0,85 & 0,88 \\
\hline$s(\hat{m})$ & 0,13 & 0,07 & 1,28 & 0,77 & 13,14 & 4,33 & 0,38 & 0,39 \\
\hline \multirow[t]{2}{*}{ CV (\%) } & 5,6 & 2,9 & 18,4 & 16,0 & 29,3 & 32,3 & 19,5 & 21,0 \\
\hline & \multicolumn{2}{|c|}{$\mathrm{Ca}\left(\mathrm{mmol}_{\mathrm{c}} \mathrm{dm}^{-3}\right)$} & \multicolumn{2}{|c|}{$\mathrm{Mg}\left(\mathrm{mmol}_{\mathrm{c}} \mathrm{dm}^{-3}\right)$} & \multicolumn{2}{|c|}{$\mathrm{H}+\mathrm{Al}\left(\mathrm{mmol}_{\mathrm{c}} \mathrm{dm}^{-3}\right)$} & \multicolumn{2}{|c|}{ V \% } \\
\hline 1 & 5327 & 1987 & 5083 & 2773 & 1747 & 2114 & 309 & 542 \\
\hline 5 & 213 & 79 & 203 & 111 & 70 & 85 & 12 & 22 \\
\hline 10 & 53 & 20 & 51 & 28 & 17 & 21 & 3 & 5 \\
\hline 15 & 24 & 9 & 23 & 12 & 8 & 9 & 1 & 2 \\
\hline 20 & 13 & 5 & 13 & 7 & 4 & 5 & 1 & 1 \\
\hline 25 & 9 & 3 & 8 & 4 & 3 & 3 & 0 & 1 \\
\hline 30 & 6 & 2 & 6 & 3 & 2 & 2 & 0 & 1 \\
\hline$\hat{m}$ & 34,1 & 35,57 & 11,17 & 12,17 & 13,37 & 28,7 & 78 & 64 \\
\hline$s$ & 9,68 & 6,17 & 3,10 & 2,49 & 2,17 & 5,13 & 5,35 & 5,80 \\
\hline$s(\hat{m})$ & 4,33 & 2,76 & 1,39 & 1,11 & 0,97 & 2,30 & 2,39 & 2,60 \\
\hline CV (\%) & 28,4 & 17,3 & 27,7 & 20,5 & 16,3 & 17,9 & 6,8 & 9,1 \\
\hline
\end{tabular}

$\hat{m}$ é a estimativa da média amostral; $s$ é o desvio-padrão; $s(\hat{m})$ é o erro-padrăo da média; $\mathrm{e} C V$ é o coeficiente de variação.

seria suficiente para avaliar a fertilidade média de uma gleba, na camada de $0-0,20 \mathrm{~m}$, e a coleta de 17 amostras simples, na camada de 0,20-0,40 m. GuARçONI et al. (2006) recomendam, para a caracterizaçáo do indivíduo solo (unidade de amostra), a coleta de 25 amostras simples, para compor uma amostra composta representativa, levando-se em conta "f" em torno de 20\%. Oliveira et al. (2007) calcularam, para os três índices de fertilidade do solo com maior variabilidade $\left(\mathrm{P}>\mathrm{Mg}^{2+}>\mathrm{K}^{+}\right)$, que a coleta de 10 a 20 amostras simples de solo para formar uma amostra composta seria suficiente para avaliar a fertilidade média de uma gleba, admitindo-se um erro de $20 \%$ em torno da média. Resultados semelhantes foram avaliados por Schlindwein e Anghinoni (2002) que estudaram solos sob plantio direto com adubação em linha e a lanço, admitindo, também, $20 \%$ de erro em torno da média. Entretanto, para avaliação do $\mathrm{P}$ no solo, os autores observaram que o número de amostras simples coletadas com trado para formar uma composta deveria ser maior que 20.

A coleta de maior número de amostras simples de solo não influi no valor médio da característica química do solo avaliada, mas aumenta a confiabilidade ou exatidão dessa média obtida pela diminuição do erro em torno da média (BARRETo et al., 1974). Quanto à variabilidade dessa média $(\mathrm{CV})$, esta tende a não se alterar quando aumenta o número de amostras simples, porque, ao mesmo tempo em que o número de amostras simples aumenta, o erro em torno da média diminui.

\section{CONCLUSÃO}

Considerando um erro aceitável de $5 \%$ a $10 \%$ para a diagnose do estado nutricional da caramboleira, a coleta de uma folha, em cada um dos quatro pontos cardeais em 21 plantas do pomar, foi suficiente para as determinaçôes químicas dos macronutrientes. Já para a análise dos micronutrientes foi necessário amostrar, no mínimo, 52 plantas de carambola.

O número de amostras simples de solo necessário para compor a amostra composta para os atributos $\mathrm{pH}$, $\mathrm{MO}, \mathrm{P}, \mathrm{K}, \mathrm{Ca}, \mathrm{Mg}, \mathrm{H}+\mathrm{Al}$ e $\mathrm{V} \%$ varia com a profundidade de amostragem. Para a mesma profundidade de coleta, o aumento do número de amostras simples de solo reduz o erro porcentual na estimativa da média desejada, permitindo a recomendaçáo de 14 e 17 subamostras, respectivamente, para as camadas de $0-0,20 \mathrm{~m}$ e $0,20-0,40$ $\mathrm{m}(\mathrm{f}=20 \%)$. 


\section{REFERÊNCIAS}

ALVAREZ V.V.H.; GUARÇONI M., A. Variabilidade horizontal da fertilidade do solo de uma unidade de amostragem em sistema de plantio direto. Revista Brasileira Ciência do Solo, v.27, p.297310, 2003.

AMARO FILHO, J.; NEGREIROS, R.F.D.; ASSIS JÚNIOR, R.N.; MOTA, J.C.A. Amostragem e variabilidade espacial de atributos físicos de um Latossolo Vermelho em Mossoró, RN. Revista Brasileira Ciência do Solo, v.31, p.415-422, 2007.

BATAGLIA, O.C.; FURLANI, A.M.C.; TEIXEIRA, J.P.F; FURLANI, P.R.; GALLO, J.R. Métodos de análise química de plantas. Campinas: Instituto Agronômico, 1983. (Boletim Técnico, 78)

BARRETO, A.C.; NOVAIS, R.F.; BRAGA, J.M. Determinação estatística do número de amostras simples de solo por área para avaliação de sua fertilidade. Revista Ceres, v.21, p.142-147, 1974.

CRANE, J.H. Tropical Fruits. Florida: Institute of food and Agricultural Science, University of Florida, 1998. v.1. CD-ROM

DAHIYA, S.S.; JOON, M.S. Variation in mineral composition of leaves of guava cultivar L-49 as affected by sample size. Crop Research, v.9, p.121-122, 1995.

DONADIO, L.C.; SILVA, J.A.A.; ARAÚJO, P.R.S., PRADO, R.M. Caramboleira (Averrhoa carambola L.) Jaboticabal: Sociedade Brasileira de Fruticultura, 2001. 81p.

EMBRAPA - EMPRESA BRASILEIRA DE PESQUISA AGROPECUÁRIA. Manual de análises químicas de solos, plantas e fertilizantes. Brasília: Embrapa Comunicação para Transferência de Tecnologia, 1999a. 370p.

EMBRAPA - EMPRESA BRASILEIRA DE PESQUISA AGROPECUÁRIA. Sistema Brasileiro de Classificaçáo de Solos. Rio de Janeiro: Centro Nacional de Pesquisa de Solos, 1999b. 412p.

FOY, C.D. Manganese and plants. In: FOY, C.D. Manganese. Washington: National Academy of Sciences, 1973. p.51-76.

GUARÇONI M.A.; ALVAREZ V.V.H.; NOVAIS, R.F.; CANTARUTTI, R.B.; LEITE, H.G.; FREIRE, F.M. Definição da dimensão do indivíduo solo e determinação do número de amostras simples necessário à sua representação. Revista Brasileira Ciência do Solo, v.30, p.943-954, 2006.

GUARÇONI M.A.; ALVAREZ V.V.H.; NOVAIS, R.F.; CANTARUTTI, R.B.; LEITE, H.G.; FREIRE, F.M. Diâmetro de trado necessário à coleta de amostras num Cambissolo sob plantio direto ou sob plantio convencional antes ou depois da araçáo. Revista Brasileira de Ciência do Solo, v.31, p.947-959, 2007.

HOLLAND, D.A.; LITTLE, R.C.; ALLEN, M.; DERMOTT, W. Soil and leaf sampling in apple orchards. Journal of Horticultural Science, v.42, p.403-417, 1967.

JAMES, D.W.; WELLS, K.L. Soil sample collection and handling: technique based on source and degree of field variability. In: WESTERMAN, R.L. (Ed.). Soil testing and plant analysis.
Madison: Soil Science Society of America, 1990. p.25-44. (SSSA Book Series, 3)

KNIGHT, P.J. Fertilizer practice in New Zealand forest nurseries. Journal Forestry Science, v.8, p.351-368, 1978.

LAMB, D. Variations in the foliar concentrations of macro and micro elements in a fast-growing tropical eucalypt. Plant and Soil, v.45, p. 477-492. 1976.

MARSCHNER, H. Mineral nutrition of higher plants. 2nd ed. San Diego: Academic, 1995. 902p

MAUSBACH, M.J.; WILDING, L.P. Spatial variabilities of soils and landforms. Madison: Soil Science Society of America, 1991. 270p. (SSSA Special Publication, 28)

MEAD, D.J. Diagnosis of nutrient deficiencies in plantations. In: BOWEN, G.D.; NAMBIAR, E.K. (Ed.). Nutrition of plantation forests. London: Academic Press, 1984. cap.10, p.259-291.

MONTESINOS, M.G.; CÁMARA, J.M.F.; TOMÉ, J.M.; CÁMARA, M.A.O.; GARCÍA, F.H.; VICENTE, R.M.; DÍEZ, M.B. Número de submuestras necessarias para la obtención de una muestra de suelo representativa. Agrícola Vergel, v.241, p.9-18, 2002.

OLIVEIRA, F.H.T; ARRUDA, J.A.; SILVA, I.F.; ALVES, J.C. Amostragem para avaliação da fertilidade do solo em função do instrumento de coleta das amostras e de tipos de preparo do solo. Revista Brasileira Ciência do Solo, v.31, p.973-983, 2007.

PAVAN, M.A; BINGHAM, F.T. Toxidez de metais em plantas. I. Caracterizaçáo de toxidez de manganês em cafeeiros. Pesquisa Agropecuária Brasileira, v.16, p.825-821, 1981.

PIMENTEL-GOMEZ, F.; GARCIA, C.H. Estatística aplicada a experimentos agronômicos e florestais: exposição com exemplos e orientaçôes para uso de aplicativos. Piracicaba: FEALQ, 2002. $309 \mathrm{p}$.

PRADO, R.M.; NATALE, W. Leaf sampling in carambola trees. Fruits, v.59, p.281-289, 2004.

RAIJ, B. van; ANDRADE, J.C.; CANTARELLA H.; QUAGGIO, J.A. Análise química para avaliação da fertilidade de solo tropicais. Campinas: Instituto Agronômico, 2001, 285p.

RAIJ, B. van; CANTARELLA, H.; QUAGGIO, J.A.; FURLANI, A.M.C. (ed.). Recomendaçôes de adubação e calagem para o Estado de São Paulo. 2.ed. Campinas: IAC, 1997. p.121-125. (Boletim Técnico, 100)

ROSSETTI, A.G.; ALMEIDA, J.I.L.; PARENTE, J.I.G.; BARROS, L.M. Tamanho ótimo de parcela para experimentos com cajueiro comum. Revista Brasileira de Fruticultura, v.13, p.117$122,1991$.

ROSSETTI, A.G.; BARROS, L.M.; ALMEIDA, J.I.L. Tamanho ótimo de parcelas para experimentos de campo com cajueiro anão precoce. Pesquisa Agropecuária Brasileira, v.31, p.843-852, 1996.

ROZANE, D.E.; NATALE, W.; PRADO, R.M.; BARBOSA, J.C. Amostragem para diagnose do estado nutricional de 
mangueiras. Revista Brasileira de Fruticultura, v.29, p.371-376, 2007.

ROZANE, D.E.; NATALE, W.; PRADO, R.M.; BARBOSA, J.C. Tamanho da amostra foliar para avaliação do estado nutricional de goiabeiras com e sem irrigação. Revista Brasileira de Engenharia Agrícola e Ambiental, v.13, p.233-239, 2009.

SCHLINDWEIN, J.A.; ANGHINONI, I. Tamanho da subamostra e representatividade da fertilidade do solo no sistema plantio direto. Ciência Rural, v.32, p.963-968, 2002.

SCHLINDWEIN, J.A.; ANGHINONI, I. Variabilidade horizontal de atributos de fertilidade e amostragem do solo no sistema de plantio direto. Revista Brasileira de Ciência do Solo, v.24, p.85-91, 2000.

SILVEIRA, P.M.; ZIMMERMANN, F.J.P.; SILVA, S.C.; CUNHA, A.A. Amostragem e variabilidade espacial de características químicas de um Latossolo submetido a diferentes sistemas de preparo. Pesquisa Agropecuária Brasileira, v.35, p.2057-2064, 2000.

SODRÉ, G.A.; MARROCOS, P.C.L.; CHEPOTE, R.E.; PACHECO, R.G. Uso do desvio padrão para estimativa do tamanho da amostra de plantas de cacau (Theobroma cacao L.) em estudos de nutrição. Agrotrópica, v.13, p.145-150, 2001.

THOMPSON, S.K. Sampling. New York: John Wiley, 1992. 343p. 